\title{
DIAGONAL PRIKRY EXTENSIONS
}

\author{
JAMES CUMMINGS AND MATTHEW FOREMAN
}

\section{INTRODUCTION}

It is a well-known phenomenon in set theory that problems in infinite combinatorics involving singular cardinals and their successors tend to be harder than the parallel problems for regular cardinals. Examples include the behaviour of cardinal exponentiation, the extent of the tree property, the extent of stationary reflection, and the existence of non-free almost-free abelian groups. The explanation for this phenomenon lies in inner model theory, in particular core models and covering lemmas. If $W$ is an inner model of $V$ then

(1) $W$ strongly covers $V$ if every uncountable set of ordinals is covered by a set of the same $V$-cardinality lying in $W$.

(2) $W$ weakly covers $V$ if $W$ computes the successor of every $V$ singular cardinal correctly.

Strong covering implies weak covering.

In inner model theory there are many theorems of the general form "if there is no inner model of large cardinal hypothesis $X$ then there is an $L$-like inner model $K_{X}$ which $Y$ covers $V$ ". Here the $L$-like properties of $K_{X}$ always include GCH and Global Square. Examples include

(1) $X$ is " $0^{\sharp}$ exists", $K_{X}$ is $L, Y$ is "strongly".

(2) $X$ is "there is a measurable cardinal", $K_{X}$ is the Dodd-Jensen core model, $Y$ is "strongly".

(3) $X$ is "there is a Woodin cardinal", $K_{X}$ is the core model for a Woodin cardinal, $Y$ is "weakly".

If $V$ is strongly covered by an inner model with $\mathrm{GCH}$ then the $\mathrm{SCH}$ holds in $V$. If $V$ is weakly covered by a model with Global Square then $\square_{\kappa}$ holds in $V$ for every $V$-singular cardinal $\kappa$, and this also exerts a strong influence on the combinatorics of $\kappa$ and $\kappa^{+}$; for example there is a special $\kappa^{+}$-Aronszajn tree. and there is a non-reflecting stationary set in $\kappa^{+}$.

First author partially supported by NSF Grants DMS-0400982 and DMS0654046 .

Second author partially supported by NSF Grants DMS-0400887 and DMS0701030 . 
Research on problems involving singular cardinals has given birth to the field of singular cardinal combinatorics. For the reasons we have discussed, the combinatorics of singular cardinals is closely bound up with large cardinals and $L$-like combinatorial principles, and involves many questions of consistency and independence. This is by no means the whole story: working in ZFC set theory Shelah has built a sophisticated theory about the power sets of singular cardinals (PCF theory), which implies for example that some consequences of $\mathrm{GCH}$ involving singular cardinals are outright theorems of ZFC.

In a series of papers with Magidor $[4,6,7,5]$ we explored the intricate web of relations between PCF theory, combinatorial principles such as square, large cardinals, stationary reflection, and forcing axioms. One of our guiding ideas was that certain objects in set theory are "canonical invariants" whose definitions may depend on the Axiom of Choice but which are independent of the choices made.

Our prior results include a PCF analysis of the generic extension by Prikry forcing (which makes a measurable cardinal into a singular cardinal of cofinality $\omega$ ), and a number of results in which non-reflecting objects of various kinds (including non-reflecting stationary sets) are constructed from PCF theoretic hypotheses. In this paper we continue this line of research.

We will prove some results in singular cardinal combinatorics, mostly by analysing the generic extensions produced by "diagonal" versions of Prikry forcing. The forcing poset used in Theorem 2.14 was introduced by Gitik and Sharon [14]. The forcing poset used in Theorem 4.19 and Remark 5.4 was introduced by Gitik and Magidor [13], and was put in the simplified form which we use here by Gitik [12].

Most of our results are connected with Shelah's PCF theory, in particular the concepts of good scale and very good scale. Let $\lambda$ be a singular cardinal, and let $A$ be an unbounded subset of $\lambda$ which consists of regular cardinals and has order type $\operatorname{cf}(\lambda)$. Then a scale of length $\lambda^{+}$in $\prod A$ is a sequence of length $\lambda^{+}$of elements of $\prod A$ which is increasing and cofinal in the eventual domination ordering. If such a scale exists we will say that $A$ carries a scale of length $\lambda^{+}$; one of the basic results in PCF theory is Shelah's theorem that for every singular cardinal $\lambda$ there is at least one $A$ which carries a scale of length $\lambda^{+}$.

A scale $\left\langle f_{\beta}: \beta<\lambda^{+}\right\rangle$of length $\lambda^{+}$in $\prod A$ is called good (resp. very good) if there is a club set $F \subseteq \lambda^{+}$, such that for every $\alpha \in F$ with $\operatorname{cf}(\lambda)<\operatorname{cf}(\alpha)$ there exist an unbounded (resp. club) set $E \subseteq \alpha$ and an element $\bar{\kappa} \in A$ such that $\left\langle f_{\beta}(\kappa): \beta \in E\right\rangle$ is strictly increasing for all $\kappa \in A$ with $\kappa>\bar{\kappa}$. 
For a detailed discussion of such scales and their applications, we refer the reader to the papers [9] and [4].

The existence of such scales is closely connected with other important phenomena in singular cardinal combinatorics. We briefly review some of the main facts.

Let $\lambda$ be singular and let $\mathcal{A}$ be the set of $A \subseteq \lambda$ such that $A$ consists of regular cardinals, $o t(A)=\operatorname{cf}(\lambda)$, and $A$ carries a scale of length $\lambda^{+}$.

(1) If $\square_{\lambda}$ holds then every $A \in \mathcal{A}$ carries a very good scale [4].

(2) If the weak square principle $\square_{\lambda}^{*}$ (or even the weaker approachability property) holds then every $A \in \mathcal{A}$ carries a good scale $[4]$.

(3) It is consistent that $\square_{\lambda}^{*}$ holds while no $A \in \mathcal{A}$ carries a very good scale [4].

(4) If the Chang conjecture $\left(\lambda^{+}, \lambda\right) \rightarrow\left(\operatorname{cf}(\lambda)^{+}, \operatorname{cf}(\lambda)\right)$ holds, then no $A \in \mathcal{A}$ carries a good scale [9].

(5) If $A \in \mathcal{A}$ and $A$ carries a good scale, then all scales of length $\lambda^{+}$in $\prod A$ are good [9].

Notice in particular that in statements $1-4$, all $A \in \mathcal{A}$ are behaving in the same way. In statement 5 all scales on a particular set $A \in \mathcal{A}$ are behaving in the same way.

In their paper "On SCH and the approachability property" [14] Gitik and Sharon solved several questions about singular cardinals.

- Woodin asked whether the failure of the Singular Cardinals Hypothesis $(\mathrm{SCH})$ at a singular cardinal $\kappa$ implies that Jensen's weak square principle $\square_{\kappa}^{*}$ holds.

- Cummings, Foreman and Magidor [4] asked whether the existence of a a very good scale of length $\kappa^{+}$implies that $\square_{\kappa}^{*}$ holds.

In the paper [14] a model is constructed in which there is a singular strong limit $\kappa$ of cofinality $\omega$ such that

(1) Some cofinal subset of $\kappa$ carries a very good scale of length $\kappa^{+}$.

(2) The weak square $\square_{\kappa}^{*}$ fails (in fact the weaker approachability property fails).

The model can be built either with $2^{\kappa}=\kappa^{+}$or $2^{\kappa}>\kappa^{+}$.

In Theorem 2.14, we prove that in the model of [14] there is a cofinal set of regular cardinals in $\kappa$ which has order type $\omega$ and carries a nongood scale of length $\kappa^{+}$. This provides an alternative proof that the approachability property fails in that model. Theorem 2.14 gives the first example known to us of a model where some cofinal subsets of a singular cardinal carry good scales, while others do not. At the end of Section 2 we discuss some related work and some open questions. 
As we mentioned already, if $A \subseteq \lambda$ and $A$ carries a good scale then all scales of length $\lambda^{+}$in $\prod A$ are good. By contrast Theorem 3.1 shows that this is not the case for very good scales. In fact we show that consistently there can be a set $A$ which carries two scales, one of which is very good and the other is very far from being very good.

Theorem 4.19 is concerned with very strong large cardinal axioms at the level of "rank into rank embeddings". Recall that given any elementary embedding $j: V \rightarrow M$ or $j: V_{\mu} \rightarrow V_{\mu}$ we may define the critical sequence by $\kappa_{0}=\operatorname{crit}(j)$ and $\kappa_{n+1}=j\left(\kappa_{n}\right)$ for all $n<\omega$. In the following discussion we let $\lambda$ be $\sup _{n} \kappa_{n}$. Kunen's celebrated inconsistency result [17] implies (Corollary 23.14 b in Kanamori's book [16]) that if $j: V \rightarrow M$ then $V_{\lambda+1} \nsubseteq \subseteq M$. We consider the axiom asserting "there is $j: V \rightarrow M$ with $V_{\lambda} \subseteq M$ ". Using an extender based Prikry forcing $[13,12]$ we show that this axiom is consistent with $2^{\lambda}>\lambda^{+}$. The proof is atypical among proofs that Prikry-style forcings preserve large cardinals in that we will be extending an elementary embedding from the ground model rather than an iterate of such an embedding.

Remark 5.4 also hinges on a PCF analysis. In his thesis [24] Assaf Sharon produced a model in which $\mathrm{SCH}$ fails at a singular strong limit cardinal $\kappa$ of cofinality $\omega$, and every stationary subset of $\kappa^{+}$reflects. The starting point is a model built by extender based Prikry forcing, but it can only be the starting point because Sharon shows that in that sort of model there is always a non-reflecting stationary set of cofinality $\omega$ points in $\kappa^{+}$. In Remark 5.4 we show that his construction can be viewed in a PCF theoretic light: to be a bit more explicit we show that extender based Prikry forcing adds a good scale in a canonical way, and we identify Sharon's non-reflecting set with a natural PCF theoretic object.

\section{Scales in the Gitik-Sharon MOdel}

We start by sketching the construction of [14], referring the reader to that paper for the details. For definiteness we will outline the version of the argument which gives a singular strong limit $\kappa$ of cofinality $\omega$ with $2^{\kappa}=\kappa^{+}$. 
- Prepare the ground model so that $\kappa$ is supercompact and $2^{\kappa}=$ $2^{\kappa^{+\omega}}=\mu$, where $\mu=\kappa^{+\omega+1}$. Arrange ${ }^{1}$ that there exist a supercompactness measure $U$ on $P_{\kappa} \mu$ and functions $F_{\beta} \in{ }^{\kappa} \kappa$ for $\beta<\mu$ such that $j_{U}\left(F_{\beta}\right)(\kappa)=\beta$.

- Project $U$ via $x \mapsto x \cap \kappa^{+n}$ to get supercompactness measures $U_{n}$ on $P_{\kappa} \kappa^{+n}$. That is to say

$$
U_{n}=\left\{A \subseteq P_{\kappa} \kappa^{+n}:\left\{x \in P_{\kappa} \mu: x \cap \kappa^{+n} \in A\right\} \in U\right\} .
$$

Each $U_{n}$ concentrates on the set $X_{n}$ of those $x \in P_{\kappa} \kappa^{+n}$ such that $\kappa_{x}={ }_{\text {def }} x \cap \kappa$ is an inaccessible cardinal and ot $(x)=\kappa_{x}^{+n}$.

- Define the main forcing $\mathbb{D}$. A condition in $\mathbb{D}$ is an $\omega$-sequence

$$
p=\left\langle x_{0}, \ldots x_{n-1}, A_{n}, A_{n+1}, \ldots\right\rangle
$$

where $x_{i} \in X_{i}$ for $i<n, A_{i} \subseteq X_{i}$ and $A_{i} \in U_{i}$ for $i \geq n$, $x_{i} \subseteq x_{i+1} \cap \kappa_{i}$, ot $\left(x_{i}\right)<\kappa_{x_{i+1}}$. We call $n$ the length and write $n=\operatorname{lh}(p)$. We call $\left\langle x_{j}: j\langle n\rangle\right.$ the lower part, and $\left\langle A_{j}: j \geq n\right\rangle$ the upper part of the condition $p$. Another condition

$$
q=\left\langle y_{0}, \ldots y_{m-1}, B_{m}, B_{m+1} \ldots\right\rangle
$$

extends $p$ if and only if $m \geq n, y_{i}=x_{i}$ for $i<n, y_{i} \in A_{i}$ for $n \leq i<m$, and $B_{i} \subseteq A_{i}$ for $i \geq m$. We say $q$ is a direct extension of $p$ and write $q \leq^{*} p$ when $q \leq p$ and $\operatorname{lh}(p)=\operatorname{lh}(q)$.

We summarise some key properties of the forcing poset $\mathbb{D}[14]$.

Fact 2.1. (1) Questions in the forcing language can be decided by direct extensions. In particular no bounded subsets of $\kappa$ are added, so $\kappa$ is preserved, as are cardinals and cofinalities below $\kappa$.

(2) The poset $\mathbb{D}$ is $\kappa^{+\omega}$-centered, in particular it has the $\kappa^{+\omega+1}$-c.c.

(3) The generic object $G$ is an infinite sequence $\left\langle y_{i}: i \in \omega\right\rangle$ such that $y_{i} \in X_{i}, y_{i} \subseteq y_{i+1}$ and $\bigcup_{i} y_{i}=\kappa^{+\omega}$. We typically write $\kappa_{i}$ for $\kappa_{y_{i}}$. Since $\bigcup_{i} y_{i}=\kappa^{+\omega}, \kappa^{+\omega}$ is collapsed to have cardinality $\kappa$, as are the cardinals $\kappa^{+n}$ for $0<n<\omega$.

\footnotetext{
${ }^{1}$ This will be true if we start with $\kappa$ supercompact, make $\kappa$ Laver indestructible [19] and then add $\mu$ many functions $\left\langle F_{\alpha}: \alpha<\mu\right\rangle$ from $\kappa$ to $\kappa$ by forcing with the poset $\mathbb{P}$ whose conditions are partial functions $p$ of cardinality less than $\kappa$ from $\mu \times \kappa$ to $\kappa$, ordered by extension. To see this perform the argument of [19] with the following modification: at the end of the proof of the Theorem (page 387, last paragraph), choose the master condition $s \in j(\mathbb{P})$ to satisfy $(*)$ and in addition to force that $j\left(F_{\alpha}\right)(\kappa)=\alpha$ for all $\alpha<\mu$. This is easy to do: if $f: \mu \times \kappa \rightarrow \kappa$ is the generic function added by $\mathbb{P}$, then $s$ is the condition in $j(\mathbb{P})$ such that $\operatorname{dom}(s)=$ $j$ " $\mu \times(\kappa+1), s(j(\alpha), \beta)=f(\alpha, \beta)$ and $s(j(\alpha), \kappa)=\alpha$ for $\alpha<\mu, \beta<\kappa$.
} 
(4) For each $n \in \omega, \kappa^{+n}=\bigcup_{i} y_{i} \cap \kappa^{+n}$. So after forcing with $\mathbb{D}, \kappa$ is a cardinal of cofinality $\omega$ and $\kappa^{+n}$ (for $\left.0<n<\omega\right)$ is an ordinal of cardinality $\kappa$ and cofinality $\omega$. In particular every $\beta<\mu$ has uncountable cofinality in $V[G]$ if and only if $\omega<\operatorname{cf}(\beta)<\kappa$ in $V$, and in this case $\mathrm{cf}^{V}(\beta)=\mathrm{cf}^{V[G]}(\beta)$.

(5) Since the cardinals $\kappa^{+i}$ for $0<i \leq \omega$ are collapsed and $\mathbb{D}$ has $\mu$ c.c. it follows that $\mu$ is preserved, and $\mu=\left(\kappa^{+\omega+1}\right)^{V}=\left(\kappa^{+}\right)^{V[G]}$.

(6) If $\left\langle A_{i}: i<\omega\right\rangle \in V$ is such that $A_{i} \in U_{i}$ for all $i$ then $y_{n} \in A_{n}$ for all large $n$ (in fact this characterises genericity).

(7) If $A \in V[G]$ is a set of ordinals such that ot $(A)=\beta$ where $\omega<\beta=c f^{V}(\beta)<\kappa$, then there is an unbounded $B \subseteq A$ with $B \in V$.

As usual when working with Prikry forcing, the main technical tool for analysing $\mathbb{D}$ is a suitable diagonal intersection lemma. The proof is an application of the normality of the measures $U_{n}$.

Lemma 2.2 (Diagonal intersection lemma for $\mathbb{D}$ ). Let $L P$ be the set of lower parts and let $\left\langle A_{s}: s \in L P\right\rangle$ be an LP-indexed family of upper parts such that $s \frown A_{s} \in \mathbb{D}$ for all $s$.

Then there exists a sequence $\left\langle B_{0}, B_{1} \ldots\right\rangle$ such that for every $n$

(1) $B_{n} \in U_{n}$.

(2) For every lower part $s=\left\langle x_{0}, \ldots x_{n-1}\right\rangle$ of length $n$, every extension of the condition $\left\langle x_{0}, \ldots x_{n-1}, B_{n}, B_{n+1}, \ldots\right\rangle$ is compatible with $s \frown A_{s}$.

Such a sequence $\left\langle B_{0}, B_{1} \ldots\right\rangle$ is called a diagonal intersection of the family $\left\langle A_{s}: s \in L P\right\rangle$.

Remark 2.3. The forcing poset $\mathbb{D}$ is a sort of diagonal version of the supercompact Prikry forcing of Magidor [21]. We briefly review this forcing: if $W$ is a supercompactness measure on $P_{\kappa} \nu$ for some $\nu>\kappa$ then conditions in the supercompact Prikry forcing $\mathbb{P}_{W}$ have the form $\left(x_{0}, \ldots x_{n-1}, A\right)$ where $x_{i} \in P_{\kappa} \nu, A \in W, x_{i} \subseteq x_{i+1}$ and ot $\left(x_{i}\right) \in x_{i+1} \cap \kappa$. The ordering is the usual Prikry-style one. This forcing satisfies properties analogous to those listed in Fact 2.1. In particular no bounded subsets of $\kappa$ are added, and all cardinals in the interval $[\kappa, \nu]$ have cofinality $\omega$ and cardinality $\kappa$ in the extension by $\mathbb{P}_{W}$. We can view $\mathbb{D}$ as interpolated between $\mathbb{P}_{U}$ and the $\mathbb{P}_{U_{i}}$, more precisely:

(1) Let $X$ be the set of $x \in P_{\kappa} \kappa^{+\omega+1}$ such that $x \cap \kappa^{+i} \in X_{i}$ for all $i$. It is easy to see that $X \in U$. If we force with $\mathbb{P}_{U}$ below the condition $(X)$ and $\left\langle z_{i}: i<\omega\right\rangle$ is the resulting generic sequence then $\left\langle z_{i} \cap \kappa^{+i}: i<\omega\right\rangle$ is generic for $\mathbb{D}$. 
(2) If $\left\langle y_{i}: i<\omega\right\rangle$ is generic for $\mathbb{D}$, then for each $n<\omega$ the sequence $\left\langle y_{i} \cap \kappa^{+n}: i<\omega\right\rangle$ is generic for $\mathbb{P}_{U_{n}}$.

Working in $V[G]$ where $G$ is $\mathbb{D}$-generic, we will describe two cofinal $\omega$-sequences in $\kappa$ such that one carries a very good scale and the other carries no good scale. The very good scale was defined by Gitik and Sharon [14].

Our main tool for analysing scales in the extension by $\mathbb{D}$ is the following result, which is a direct descendent of the analysis of scales in a Prikry extension [15]. We recall our convention that $\kappa_{n}=\kappa_{y_{n}}=y_{n} \cap \kappa$, where $\left\langle y_{i}: i<\omega\right\rangle$ is a $\mathbb{D}$-generic sequence.

Lemma 2.4. (Bounding lemma) Let $\eta: \omega \rightarrow \kappa$ be such that $\eta(n)$ is a successor ordinal with $\eta(n)\rangle n$. Let $G=\left\langle y_{i}: i<\omega\right\rangle$ be $\mathbb{D}$ generic. Let $h \in V[G]$ with $h \in \prod_{n} \kappa_{n}^{+\eta(n)}$. Then there exists a sequence $\left\langle H_{n}: n<\omega\right\rangle \in V$ such that $\operatorname{dom}\left(H_{n}\right)=X_{n}, H_{n}(x)<\kappa_{x}^{+\eta(n)}$ for all $x \in X_{n}$, and $h(n)<H_{n}\left(y_{n}\right)$ for all large $n$.

Proof. Let $\dot{h}$ be a name and let $p=\left\langle x_{0}, \ldots x_{n}, A_{n+1}, \ldots\right\rangle$ be a condition forcing that $\dot{h} \in \prod_{n} \kappa_{n}^{+\eta(n)}$.

Let $t$ be a lower part extending $\left\langle x_{0}, \ldots x_{n}\right\rangle$, say $t=\left\langle x_{0}, \ldots x_{m}\right\rangle$ for some $m \geq n$. Then the condition $q$ with lower part $t$ and trivial upper part determines the value of $\kappa_{m}$, namely it forces it to be $\kappa_{x_{m}}$. So $q$ forces that $\dot{h}(m)<\kappa_{x_{m}}^{+\eta(m)}$, where we note that $\kappa_{x_{m}}^{+\eta(m)}<\kappa$ because $\kappa_{x_{m}}<\kappa$ and $\eta(m)<\kappa$.

By the direct extension property and the $\kappa$-completeness of the measures $U_{j}$ we may therefore find an upper part $A_{t}$ such that $t^{\frown} A_{t}$ determines the value of $\dot{h}(m)$, say $t^{\frown} A_{t} \Vdash \dot{h}(m)=g(t)$. We now form the diagonal intersection $\left\langle B_{0}, B_{1}, \ldots\right\rangle$, and build a direct extension $p^{\prime}=\left\langle x_{0}, \ldots x_{n-1}, A_{n}^{\prime}, A_{n+1}^{\prime} \ldots\right\rangle$ by setting $A_{m}^{\prime}=A_{m} \cap B_{m}$ for $m \geq n$.

By construction $p^{\prime}$ forces that $\dot{h}(m)=g\left(\left\langle y_{0}, \ldots y_{m}\right\rangle\right)$ for all $m \geq n$, where $\left\langle y_{0}, y_{1}, \ldots\right\rangle$ is the generic $\omega$-sequence added by $\mathbb{D}$. For each $m$ and each $z \in X_{m}$ we have $|z|=\kappa_{z}^{+m}$, and so there are at most $\kappa_{z}^{+m}$ many lower parts $\left\langle z_{0}, \ldots z_{m}\right\rangle$ with $z_{m}=z$; accordingly we define

$H_{m}(z)=\sup \left\{g\left(\left\langle z_{0}, \ldots z_{m}\right\rangle\right)+1:\left\langle z_{0}, \ldots z_{m}\right\rangle\right.$ a lower part with $\left.z_{m}=z\right\}$

Then $H_{m}(z)<\kappa_{z}^{+\eta(m)}$ because $\eta(m)>m$, and $p^{\prime}$ forces that $\dot{h}(m)<$ $H_{m}\left(y_{m}\right)$ for all $m \geq n$.

2.1. The bad scale. Recall that in $V$ we have $2^{\kappa^{+\omega}}=\kappa^{+\omega+1}=\mu$. Working in $V$ we fix a scale $\left\langle G_{\beta}: \beta<\mu\right\rangle$ in $\prod_{n} \kappa^{+n+1}$. Standard arguments due to Shelah (see for instance [3] or [9] for the details) show that owing to the supercompactness of $\kappa$ there are stationarily 
many $\alpha \in \mu \cap \operatorname{cof}(<\kappa)$ which are not good points. For each $n$ and each $\eta<\kappa^{+n+1}$ fix a function $F_{n}^{\eta}$ with domain $X_{n}$ such that $F_{n}^{\eta}(x)<\kappa_{x}^{+n+1}$ for all $x \in X_{n}$, and $\left[F_{n}^{\eta}\right]_{U_{n}}=\eta$. This is possible because $U l t\left(V, U_{n}\right)$ is closed under $\kappa^{+n}$-sequences, so that $\kappa^{+n+1}=\left(\kappa^{+n+1}\right)^{U l t\left(V, U_{n}\right)}$.

Now let $G=\left\langle y_{i}: i<\omega\right\rangle$ be $\mathbb{D}$-generic. Working in $V[G]$ we define a sequence of functions $\left\langle g_{\beta}: \beta<\mu\right\rangle$ in $\prod_{n} \kappa_{n}^{+n+1}$, by setting $g_{\beta}(n)=$ $F_{n}^{G_{\beta}(n)}\left(y_{n}\right)$.

Lemma 2.5. In $V[G]$ the sequence $\left\langle g_{\beta}: \beta<\mu\right\rangle$ forms a scale in $\prod_{n} \kappa_{n}^{+n+1}$, and this scale is not good.

Proof. We work in the model $V[G]$.

Claim 2.6. The sequence $\left\langle g_{\beta}: \beta<\mu\right\rangle$ is increasing modulo finite.

Proof. Let $\beta<\gamma<\mu$, then for all large $n$ we have $G_{\beta}(n)<G_{\gamma}(n)$, so that $F_{n}^{G_{\beta}(n)}<_{U_{n}} F_{n}^{G_{\gamma}(n)}$. If we let $A_{n}=\left\{x \in X_{n}: F_{n}^{G_{\beta}(n)}(x)<\right.$ $\left.F_{n}^{G_{\gamma}(n)}(x)\right\}$ then $y_{n} \in A_{n}$ for all large $n$, so by definition $g_{\beta}(n)<g_{\gamma}(n)$ for all large $n$.

Claim 2.7. The sequence $\left\langle g_{\beta}: \beta<\mu\right\rangle$ forms a scale in $\prod_{n} \kappa_{n}^{+n+1}$

Proof. Let $h \in V[G]$ with $h \in \prod_{n} \kappa_{n}^{+n+1}$. By the Bounding Lemma we obtain $\left\langle H_{n}: n<\omega\right\rangle \in V$ so that $h(n)<H_{n}\left(y_{n}\right)$ for all large $n$. The map $n \mapsto\left[H_{n}\right]_{U_{n}}$ is in $V$ and is an element of $\prod_{n} \kappa^{+n+1}$, and since the $G_{\beta}$ form a scale in $\prod_{n} \kappa^{+n+1}$ we may find $\beta$ such that $\left[H_{n}\right]_{U_{n}}<G_{\beta}(n)$ for all large $n$. Since $G_{\beta}(n)=\left[F_{n}^{G_{\beta}(n)}\right]_{U_{n}}$, we see that if $A_{n}=\left\{x \in X_{n}: H_{n}(x)<F_{n}^{G_{\beta}(n)}(x)\right\}$ then $A_{n} \in U_{n}$ for all large $n$. Then $y_{n} \in A_{n}$ for all large $n$, so $h(n)<H_{n}\left(y_{n}\right)<g_{\beta}(n)$ for all large $n$.

Claim 2.8. In $V[G]$ the scale $\left\langle g_{\beta}: \beta<\mu\right\rangle$ is not good.

Proof. Suppose that in $V[G]$ we have a point $\alpha<\mu$ where $\omega<c f(\alpha)<$ $\kappa$ such that $\alpha$ is good for the scale $\left\langle g_{\beta}: \beta<\mu\right\rangle$. By Fact $2.1 \omega<$ $c f(\alpha)<\kappa$ in $V$ also. We will show that $\alpha$ is good in $V$ for the scale $\left\langle G_{\beta}: \beta<\mu\right\rangle$. Since stationary sets in $\kappa^{+\omega+1}$ are preserved by the $\kappa^{+\omega_{-}}$ centered poset $\mathbb{D}$, and the scale $\left\langle G_{\beta}: \beta<\mu\right\rangle$ has stationarily many points which are not good, this is enough to show that in $V[G]$ the scale $\left\langle g_{\beta}: \beta<\mu\right\rangle$ has stationarily many points which are not good.

By Fact 2.1 every unbounded subset of $\alpha$ in $V[G]$ contains an unbounded set from the ground model. So we may assume that there exist $A \in V$ unbounded in $\alpha$ and $m$ witnessing that $\alpha$ is good for the scale $\left\langle g_{\beta}: \beta<\mu\right\rangle$, that is to say $\left\langle g_{\beta}(t): \beta \in A\right\rangle$ is strictly increasing for all $t \geq m$. 
We choose a condition $p=\left\langle x_{0}, \ldots x_{n-1}, A_{n} \ldots\right\rangle$ forcing that " $A$ and $m$ witness that $\alpha$ is good for the scale $\left\langle\dot{g}_{\beta}: \beta<\mu\right\rangle$ ". It must be the case that for every $t \in \omega$ with $t \geq m, n$ there are $U_{t}$-many $x \in A_{t}$ such that the sequence $\left\langle F_{t}^{G_{\beta}(t)}(x): \beta \in A\right\rangle$ is strictly increasing: for otherwise we can extend $p$ to $q$ forcing that " $\left\langle\dot{g}_{\beta}(t): \beta \in A\right\rangle$ is not strictly increasing".

Let $\beta, \gamma \in A$ with $\beta<\gamma$ and let $t \geq m, n$; then $\left\{x \in X_{t}: F_{t}^{G_{\beta}(t)}(x)<\right.$ $\left.F_{t}^{G_{\gamma}(t)}(x)\right\} \in U_{t}$, so $G_{\beta}(t)=\left[F_{t}^{G_{\beta}(t)}\right]_{U_{t}}<\left[F_{t}^{G_{\gamma}(t)}\right]_{U_{t}}=G_{\gamma}(t)$. So $\left\langle G_{\beta}(t)\right.$ : $\beta \in A\rangle$ is strictly increasing for every $t \geq m, n$. By definition it follows that in $V$ the ordinal $\alpha$ is a good point for the scale $\left\langle G_{\beta}: \beta<\mu\right\rangle$.

This concludes the proof of Lemma 2.5.

Remark 2.9. Lemma 2.5 gives an alternative proof that the approachability property fails in the model of Gitik and Sharon.

2.2. The very good scale. For completeness we describe the very good scale defined by Gitik and Sharon in the extension by $\mathbb{D}$. Recall that we prepared $V$ so that there exist functions $F_{\beta}: \kappa \rightarrow \kappa$ such that $j_{U}\left(F_{\beta}\right)(\kappa)=\beta$ for every $\beta<\mu$. Working in $V[G]$, we define a sequence $\left\langle f_{\beta}: \beta<\mu\right\rangle$ of functions in $\prod_{n} \kappa_{n}^{+\omega+1}$ by setting $f_{\beta}(n)=F_{\beta}\left(\kappa_{n}\right)$.

Lemma 2.10. In $V[G]$ the sequence $\left\langle f_{\beta}: \beta<\mu\right\rangle$ forms a very good scale in $\prod_{n} \kappa_{n}^{+\omega+1}$.

Proof. We work in the model $V[G]$.

Claim 2.11. The sequence $\left\langle f_{\beta}: \beta<\mu\right\rangle$ is increasing modulo finite.

Proof. Let $\beta<\gamma<\mu$. Then each $U_{n}$ concentrates on the set of those $x \in X_{n}$ such that $F_{\beta}\left(\kappa_{x}\right)<F_{\gamma}\left(\kappa_{x}\right)$, and so as in the proof of Lemma 2.5 genericity implies that $f_{\beta}(n)<f_{\gamma}(n)$ for all large $n$.

Claim 2.12. The sequence $\left\langle f_{\beta}: \beta<\mu\right\rangle$ forms a scale in $\prod_{n} \kappa_{n}^{+\omega+1}$.

Proof. Let $h \in \prod_{n} \kappa_{n}^{+\omega+1}$, and apply the Bounding Lemma to find a sequence $\left\langle H_{n}: n<\omega\right\rangle \in V$ with $H_{n}(x)<\kappa_{x}^{+\omega+1}$ for all $x \in X_{n}$, and $h(n)<H_{n}\left(y_{n}\right)$ for all large $n$. Now define a map $H_{n}^{*}$ with domain $P_{\kappa} \mu$ by $H_{n}^{*}(y)={ }_{\text {def }} H_{n}\left(y \cap \kappa^{+n}\right)$, and let $\beta_{n}=\left[H_{n}^{*}\right]_{U}$. Since $H_{n}^{*}(y)<\kappa_{y}^{+\omega+1}$ for all $y$, it follows by normality that $\beta_{n}<\kappa^{+\omega+1}$, so we may choose $\beta<\kappa^{+\omega+1}$ with $\beta_{n}<\beta$ for all $n$. It is easy to see that each $U_{n}$ concentrates on the set of those $x \in X_{n}$ such that $H_{n}(x)<F_{\beta}\left(\kappa_{x}\right)$, and so by genericity we get that $h\left(y_{n}\right)<f_{\beta}(n)$ for all large $n$.

Claim 2.13. In $V[G]$ the scale $\left\langle f_{\beta}: \beta<\mu\right\rangle$ is very good. 
Proof. As in the proof of Lemma 2.5, we only need to consider ordinals $\alpha<\mu$ where $\omega<\operatorname{cf}(\alpha)<\kappa$ in $V$. Given such an $\alpha$ we fix $A \in V$ a club subset of $\alpha$ with ot $(A)<\kappa$. It is easy to see that each $U_{n}$ concentrates on the set of those $x \in X_{n}$ such that the sequence $\left\langle F_{\beta}\left(\kappa_{x}\right): \beta \in A\right\rangle$ is increasing and continuous, so by genericity for all sufficiently large $n$ the sequence $\left\langle f_{\beta}(n): \beta \in A\right\rangle$ is increasing and continuous.

This concludes the proof of Lemma 2.10.

Combining the results of Lemmas 2.5 and 2.10, we have proved the following Theorem.

Theorem 2.14. If $G$ is $\mathbb{D}$-generic then in $V[G]$

(1) The set $\left\{\kappa_{n}^{+\omega+1}: n<\omega\right\}$ carries a very good scale.

(2) The set $\left\{\kappa_{n}^{+n+1}: n<\omega\right\}$ carries a scale which is not good.

The lack of uniformity in the PCF structure of $V[G]$ which is made manifest in Theorem 2.14 seems intriguing. It is vaguely reminiscent of the situation in some models of Shelah [25, 26], where there is a stationary and co-stationary set $S \subseteq \omega_{1}$ such that $\nabla_{S}$ holds but on $S^{c}$ the universe resembles a model of Martin's Axiom.

We finish this section with some remarks and open questions. For some background (and more questions) we refer the reader to Foreman's paper [8] from the proceeding of the 2004 Banff Singular Cardinal Combinatorics meeting.

Remark 2.15. Results of the same kind as Theorem 2.14 have recently been proved in some related models.

(1) In an early version of this paper, we raised the question of whether Theorem 2.14 can be generalised to singular cardinals of uncountable cofinality. In her doctoral thesis Dima Sinapova answered this question. Sinapova [27] introduced a version of the Gitik-Sharon forcing which makes a supercompact cardinal $\kappa$ into a singular cardinal of uncountable cofinality, and showed that in her model there are cofinal sets carrying a non-good scale and a very good scale.

(2) Itay Neeman [23] used a variant of the Gitik-Sharon construction to produce a model in which the Singular Cardinals Hypothesis fails at a singular cardinal $\kappa$ of cofinality $\omega$, and $\kappa^{+}$has the tree property. This model also has both a non-good scale and a very good scale.

We finish with some questions:

(1) Are there other interesting scales of length $\kappa^{+}$in the models of [14] or [27]? 
(2) In the scale of Lemma 2.5, in which case of Shelah's Trichotomy Theorem ([3]) do the non-good points fall?

(3) What can be proved in ZFC about the extent of good scales? For example when the first PCF generator exists, does it have a maximal subset on which good scales can live?

(4) Let $\lambda$ be singular and suppose that every cofinal set of regular cardinals in $\lambda$ with order type $\operatorname{cf}(\lambda)$ carries a very good scale. Does this imply that the approachability property holds, or that there exists a $\lambda^{+}$-Aronszajn tree?

(5) There are several open problems regarding the question whether the results we have discussed can be brought down to small singular cardinals. As far as we know the following are still open:

(a) Can it be the case that $\aleph_{\omega}$ is strong limit and there exist both very good and non-good scales of length $\aleph_{\omega+1}$ ? Gitik and Sharon [14] build a model where this will hold with $\aleph_{\omega^{2}}$ in place of $\aleph_{\omega}$, also a model where these scales will exist and $p p\left(\aleph_{\omega}\right)>\aleph_{\omega+1}$.

(b) Can there exist a singular strong limit cardinal $\kappa$ which is not a cardinal fixed point, such that $2^{\kappa}>\kappa^{+}$and $\kappa^{+}$has the tree property?

\section{DEBASING A VERY GOOD SCALE}

As we mentioned in Section 1, the concept of "good scale" is quite robust. To be more precise, suppose that $\lambda$ is singular, $A$ is a set of regular cardinals which is cofinal in $\lambda$ with order type $\operatorname{cf}(\lambda)$, and $\left\langle f_{\alpha}: \alpha<\lambda^{+}\right\rangle$and $\left\langle g_{\alpha}: \alpha<\lambda^{+}\right\rangle$are both scales in $\prod A$. If we let $C$ be the set of $\gamma<\lambda^{+}$such that for every $\alpha<\gamma$ there is $\beta<\gamma$ with $f_{\alpha}<^{*} g_{\beta}$ and $g_{\alpha}<^{*} f_{\beta}$, then $C$ is a club subset of $\lambda^{+}$. It is easy to see that if $\gamma \in C$ then the properties " $\gamma$ is a good point for $\left\langle f_{\alpha}: \alpha<\lambda^{+}\right\rangle$" and " $\gamma$ is a good point for $\left\langle g_{\alpha}: \alpha<\lambda^{+}\right\rangle$" are equivalent; in particular one scale is good if and only if the other scale is good. More generally this shows that we may assign to $A$ a subset of $\lambda^{+}$which is well-defined modulo clubs and turns out to be a stationary set with a close relationship to the combinatorics of $\lambda$ and $\lambda^{+}$. This "good set" is an example of the kind of "canonical structure" which we discussed in our earlier papers [6] and [7], and is a key object in many problems about singular cardinals (see for example [22] or [2]).

The difference between the definition of good point and very good point looks quite small, but we will show in this section that the difference is quite substantial in the sense that the set of very good points 
for a scale fails to have the same "canonical" nature that we showed for the good points in the preceding paragraph. In fact we will produce an example in which the same set $A$ carries both a very good scale and a scale which has no very good points at all.

We assume for the rest of this section that $V=L$. We need some facts about the existence of square sequences and scales in $L$.

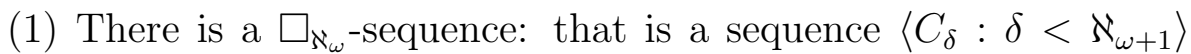
such that

(a) For every $\delta$ the set $C_{\delta}$ is club in $\delta$, with ot $\left(C_{\delta}\right)<\aleph_{\omega}$.

(b) For every $\delta$ and every $\beta \in \lim \left(C_{\delta}\right), C_{\beta}=C_{\delta} \cap \beta$.

(2) There is a partial square on $\aleph_{\omega+1} \cap \operatorname{cof}\left(\omega_{1}\right)$ : that is a sequence $\left\langle D_{\delta}: \delta \in \aleph_{\omega+1} \cap \operatorname{cof}\left(\omega_{1}\right)\right\rangle$ such that

(a) For every $\delta$ the set $D_{\delta}$ is club in $\delta$, with ot $\left(D_{\delta}\right)=\omega_{1}$.

(b) For every $\delta, \delta^{\prime}$ and every $\beta \in \lim \left(D_{\delta}\right) \cap \lim \left(D_{\delta^{\prime}}\right), D_{\delta} \cap \beta=$ $D_{\delta^{\prime}} \cap \beta$.

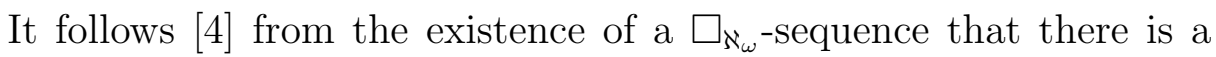
very good scale $\left\langle f_{\alpha}: \alpha<\aleph_{\omega+1}\right\rangle$ of length $\aleph_{\omega+1}$ in $\prod_{n<\omega} \aleph_{n}$. We will modify each function in this scale on a finite set to get a new sequence $\left\langle g_{\alpha}: \alpha<\aleph_{\omega+1}\right\rangle$. Since the property of being a scale is defined in terms of domination modulo finite sets, $\left\langle g_{\alpha}: \alpha<\aleph_{\omega+1}\right\rangle$ will also be a scale; we will make our alterations to ensure that $\left\langle g_{\alpha}: \alpha<\aleph_{\omega+1}\right\rangle$ has no very good points.

Fix a partition of $\omega_{1}$ into $\omega$ disjoint stationary sets, say $\left\langle S_{n}: n<\omega\right\rangle$. We define $n_{\alpha}<\omega$ for each $\alpha<\aleph_{\omega+1}$ as follows:

(1) If there is no $\delta \in \aleph_{\omega+1} \cap \operatorname{cof}\left(\omega_{1}\right)$ such that $\alpha \in \lim \left(D_{\delta}\right)$ then $n_{\alpha}=0$.

(2) If there is $\delta \in \aleph_{\omega+1} \cap \operatorname{cof}\left(\omega_{1}\right)$ such that $\alpha \in \lim \left(D_{\delta}\right)$ then let $\beta=\operatorname{ot}\left(D_{\delta} \cap \alpha\right)$, where we note that $\beta$ is independent of the choice of $\delta$. Then let $n_{\alpha}$ be the unique $n$ such that $\beta \in S_{n}$.

We now define $g_{\alpha}$ by setting $g_{\alpha}(m)=0$ for $m<n_{\alpha}, g_{\alpha}(m)=f_{\alpha}(m)$ for $m \geq n_{\alpha}$. We claim that the scale $\left\langle g_{\alpha}: \alpha<\aleph_{\omega+1}\right\rangle$ has no very good points. Suppose for a contradiction that $\beta$ is a very good point: by definition $\operatorname{cf}(\beta)>\omega$, and there exist a club set $E \subseteq \beta$ and $m<\omega$ such that $\left\langle g_{\alpha}(n): \alpha \in E\right\rangle$ is strictly increasing for all $n$ such that $m \leq n<\omega$. Let $\delta=\beta$ if $\operatorname{cf}(\beta)=\omega_{1}$, otherwise let $\delta$ be the least point in $\lim (E) \cap \operatorname{cof}\left(\omega_{1}\right)$; in either case $\operatorname{cf}(\delta)=\omega_{1}, E$ is club in $\delta$, and $\left\langle g_{\alpha}(n): \alpha \in E \cap \delta\right\rangle$ is strictly increasing. So $E \cap C_{\delta}$ is a club set in $\delta$, and hence there is a stationary set $S$ of $\alpha \in E \cap C_{\delta}$ such that ot $\left(C_{\delta} \cap \alpha\right) \in S_{m+1}$. Let $\alpha<\alpha^{\prime}$ with $\alpha, \alpha^{\prime} \in S$; by definition $n_{\alpha}=n_{\alpha^{\prime}}=$ $m+1$, so that $g_{\alpha}(m)=g_{\alpha^{\prime}}(m)=0$, but this is a contradiction because $\left\langle g_{\alpha}(m): \alpha \in E\right\rangle$ is strictly increasing and $\alpha, \alpha^{\prime} \in E$. 
We summarise the work of this section in a Theorem.

Theorem 3.1. If $V=L$ the set $\left\{\aleph_{n}: n<\omega\right\}$ carries both a very good scale, and a scale which fails to be very good at every point.

\section{FAILURE OF SCH AND RANK-INTO-RANK EMBEDDINGS}

An important theme in infinite combinatorics is the interaction between large cardinals and the continuum function. An early landmark is the result by Solovay [28] that the Singular Cardinals Hypothesis holds above a strongly compact cardinal. In this section we prove a result which shows that a large cardinal axiom of higher consistency strength has a more limited effect: more precisely we will show in Theorem 4.19 that the existence of a non-trivial $\Sigma_{1}^{1}$-elementary embedding from $V_{\lambda}$ to $V_{\lambda}$ (where $\lambda$ is a strong limit cardinal of cofinality $\omega$ ) does not impose any bound on $2^{\lambda}$.

We start by reviewing the version of extender based forcing described in section 2 of [12]. Most of the facts which we need appear in that paper and all of them are implicit there. We sketch proofs of the facts which are neither very easy nor proved explicitly in [12]. The reader can also consult Gitik's excellent survey paper [11] on Prikry-type forcing.

4.1. The extenders. We start by constructing the extenders on which the forcing is based; these are "extenders" in the broad sense of "commutative systems of ultrafilters derived from an elementary embedding". We will assume GCH for the sake of simplicity. Let $\kappa<\rho$ be regular where there is $j: V \rightarrow M$ with critical point $\kappa$ such that $j(\kappa)>\rho$ and all bounded subsets of $\rho$ are in $M$. For each $\alpha<\rho$ let $E_{\alpha}=\{X \subseteq \kappa: \alpha \in j(X)\}$, so that $E_{\alpha}$ is a $\kappa$-complete ultrafilter on $\kappa$.

We recall the Rudin-Keisler ordering on ultrafilters on $\kappa$. If $f: \kappa \rightarrow \kappa$ and $U$ is an ultrafilter on $\kappa$ then $f^{*} U={ }_{\operatorname{def}}\left\{A \subseteq \kappa: f^{-1}[A] \in U\right\}$ is also an ultrafilter on $\kappa$. Given ultrafilters $U, V$ on $\kappa$ we say that $U \leq_{R K} V$ if and only if $U=f^{*} V$ for some $f$, and in this case we say that $U$ is the RK-projection of $V$ by $f$. The relation is $\leq_{R K}$ is reflexive and transitive.

Returning to the ultrafilters $E_{\alpha}$, we choose for each $\alpha$ and $\beta$ with $E_{\alpha} \leq_{R K} \beta$ a function $\pi_{\alpha \beta}: \kappa \rightarrow \kappa$ such that $\pi_{\alpha \beta}^{*} E_{\beta}=E_{\alpha}$. For all $\alpha$ we have $E_{\alpha} \leq E_{\alpha}$, and we choose $\pi_{\alpha \alpha}=i d_{\kappa}$. The following Lemma summarises some results from [12].

Lemma 4.1. (1) For all $\alpha$ and $\beta$, if $E_{\alpha} \leq_{R K} E_{\beta}$ then $j\left(\pi_{\alpha \beta}\right)(\beta)=$ $\alpha$.

(2) For all $\alpha, \beta, \gamma$ such that $E_{\alpha} \leq_{R K} E_{\beta} \leq_{R K} E_{\gamma}, \pi_{\alpha \beta} \circ \pi_{\beta \gamma} \simeq_{E_{\gamma}} \pi_{\alpha \gamma}$. 
(3) For all $a \in[\rho]^{<\kappa}$ there are unboundedly many $\beta<\rho$ such that $E_{\alpha} \leq E_{\beta}$ for all $\alpha \in a$.

The extender $E$ is the system of measures $\left\langle E_{\alpha}: \alpha<\rho\right\rangle$ and maps $\left\langle\pi_{\alpha \beta}: E_{\alpha} \leq_{R K} E_{\beta}\right\rangle$.

Definition 4.2. We say that $a \in[\rho]^{<\kappa}$ is topped if and only if a has a maximal element and $E_{\alpha} \leq E_{\max (a)}$ for all $\alpha \in a$.

By Lemma 4.1 every small set can be made topped by adding one element.

Definition 4.3. Let a be topped with $\max (a)=\gamma$ and let $\nu<\kappa$. $\nu$ is sorted for $a$ if and only if

(1) For all $\alpha, \beta \in$ a with $E_{\alpha} \leq_{R K} E_{\beta}$ we have $\pi_{\alpha \gamma}(\nu)=\pi_{\alpha \beta}\left(\pi_{\beta \gamma}(\nu)\right)$.

(2) For all $\alpha, \beta \in$ a with $\alpha<\beta$ we have $\pi_{\alpha \gamma}(\nu)<\pi_{\beta \gamma}(\nu)$.

The following Lemma follows easily from the $\kappa$-completeness of $E_{\gamma}$.

Lemma 4.4. If $a$ is topped with $\max (a)=\gamma$ then the set of $\nu$ which are sorted for a is in $E_{\gamma}$.

We now assume we are given an increasing sequence $\kappa_{n}$ for $n<\omega$, a cardinal $\rho>\sup _{n} \kappa_{n}$, and embeddings $j_{n}: V \rightarrow M_{n}$ such that $\operatorname{crit}\left(j_{n}\right)=\kappa_{n}, j_{n}\left(\kappa_{n}\right)>\rho$, and $M_{n}$ contains all bounded subsets of $\rho$.

For each $n$ we generate an extender $E^{n}$ as above, with measures $\left\langle E_{\alpha}^{n}\right.$ : $\alpha<\rho\rangle$ and projection maps $\left\langle\pi_{\alpha \beta}^{n}: E_{\alpha} \leq_{R K} E_{\beta}\right\rangle$. Let $\kappa_{\omega}=\operatorname{def}_{\operatorname{dup}_{n} \kappa_{n}}$ and let $\vec{E}=\left\langle E_{n}: n<\omega\right\rangle$.

4.2. The basic module $\mathbb{Q}_{n}$. We will ultimately describe a forcing poset $\mathbb{Q}^{\vec{E}}$ which will add functions $f_{\alpha} \in \prod_{n} \kappa_{n}$ for every $\alpha<\rho$. We start by describing the "modules" $\mathbb{Q}^{E^{n}}$, which will be combined to produce $\mathbb{Q}^{\vec{E}}$. As we explain in more detail in subsection 4.4 below, a condition in $\mathbb{Q}^{E^{n}}$ provides some information about the values $f_{\alpha}(n)$ for $\alpha<\rho$.

Definition 4.5. The set $\mathbb{Q}^{E^{n}}$ is the disjoint union of $\mathbb{Q}_{0}^{E^{n}}$ and $\mathbb{Q}_{1}^{E^{n}}$ where

(1) $\mathbb{Q}_{1}^{E^{n}}$ is the set of partial functions from $\rho$ to $\kappa_{n}$ with support of size at most $\kappa_{\omega}$.

(2) $\mathbb{Q}_{0}^{E^{n}}$ is the set of triples $(a, A, f)$ where
(a) $a \in[\rho]^{<\kappa_{n}}$ and $a$ is topped.
(b) $A \in E_{\max (a)}^{n}$.
(c) Every $\nu \in A$ is sorted for a.
(d) $f \in \mathbb{Q}_{1}^{E^{n}}$ and $\operatorname{dom}(f) \cap a=\emptyset$. 
We will need various orderings on these sets.

\section{Definition 4.6.}

(1) Let $f, g \in \mathbb{Q}_{1}^{E^{n}}$. Then $g \leq_{n 1} f$ if and only if $g$ extends $f$.

(2) Let $(b, B, g),(a, A, f) \in \mathbb{Q}_{0}^{E^{n}}$. Then

(a) $(b, B, g) \leq_{n 0}(a, A, f)$ if and only if $b \supseteq a, g$ extends $f$ and $\pi_{\max (a) \max (b)}^{n}[B] \subseteq A$.

(b) $(b, B, g) \leq_{n 0}^{+}(a, A, f)$ if and only if $b=a, B \subseteq A$ and $g$ extends $f$.

(c) $(b, B, g) \leq_{n 0}^{++}(a, A, f)$ if and only if $b=a, B=A$ and $g$ extends $f$.

(3) Let $g \in \mathbb{Q}_{1}^{E^{n}}$ and $(a, A, f) \in \mathbb{Q}_{0}^{E^{n}}$. Then $g \leq_{n 10}(a, A, f)$ if and only if $g$ extends $f, a \subseteq \operatorname{dom}(g), g(\max (a)) \in A$, and $g(\alpha)=\pi_{\alpha \max (a)}^{n}(g(\max a))$ for every $\alpha \in a$.

(4) The orderings $\leq_{n}, \leq_{n}^{*}$ and $\leq_{n}^{* *}$ on $\mathbb{Q}^{E^{n}}$ are given by
(a) $\leq_{n}=\leq_{n 0} \cup \leq_{n 1} \cup \leq_{n 10}$
(b) $\leq_{n}^{*}=\leq_{n 0} \cup \leq_{n 1}$.
(c) $\leq_{n}^{* *}=\leq_{n 0}^{+} \cup \leq_{n 1}$.

The proof of the following Lemma is tedious but routine.

Lemma 4.7. Each of $\leq_{n}, \leq_{n}^{*}, \leq_{n}^{* *}$ is a partial ordering of $\mathbb{Q}^{E^{n}}$. The ordering $\leq_{n}^{*}$ is $\kappa_{n}$-directed closed, and the ordering $\leq_{n 0}^{++}$is $\kappa_{\omega}^{+}$-closed.

Definition 4.8. If $q=(a, A, f)$ is in $\mathbb{Q}_{0}^{E^{n}}$ and $\nu \in A$ then $q+\nu$ is the condition $g \in \mathbb{Q}_{1}^{E^{n}}$ given by $\operatorname{dom}(g)=\operatorname{dom}(f) \cup a, g\lceil\operatorname{dom}(f)=f$, $g(\alpha)=\pi_{\alpha \max (a)}^{n}(\nu)$ for all $\alpha \in a$.

The condition $q+\nu$ is the weakest $g \in \mathbb{Q}_{1}^{E^{n}}$ such that $g \leq_{n} q$ and $g(\max (a))=\nu$, and any such $g$ can be obtained by first extending the " $f$-part" of $q$ and then adding in $\nu$. We record this in a Lemma.

Lemma 4.9. Let $q=(a, A, f) \in \mathbb{Q}_{0}^{E^{n}}$ and let $g \in \mathbb{Q}_{1}^{E^{n}}$ with $g \leq_{n} q$. let $\nu=g(\max a)$. Then

(1) $g \leq_{n} q+\nu$.

(2) There is a unique $r \in \mathbb{Q}_{0}^{E^{n}}$ such that $r \leq_{n 0}^{++} q$ and $g=r+\nu$.

4.3. The main forcing. We define $\mathbb{Q}^{\vec{E}}$ to be the set of $p$ such that

(1) $p$ is an $\omega$-sequence $\left\langle p_{n}: n<\omega\right\rangle$.

(2) There is an integer $l=l(p)$ such that $p_{n}=f_{n} \in \mathbb{Q}_{1}^{E^{n}}$ for $n<l$ and $p_{n}=\left(a_{n}, A_{n}, f_{n}\right) \in \mathbb{Q}_{0}^{E^{n}}$ for $n \geq l$.

(3) $a_{n} \subseteq a_{n+1}$ for $n \geq l$.

We will need various orderings of $\mathbb{Q}^{\vec{E}}$. When we force with $\mathbb{Q}^{\vec{E}}$ we will use the partial ordering $\leq$, the other orderings are auxiliary concepts used in the analysis of the forcing extension. 
Definition 4.10. Let $p, q \in \mathbb{Q}^{\vec{E}}$.

(1) $p \leq q$ if and only if $p_{n} \leq_{n} q_{n}$ for all $n$.

(2) (Direct extension) Let $p$ and $q$ be conditions. Then $p$ is a direct extension of $q$ (and we write $p \leq{ }^{*} q$ ) if and only if $p \leq q$ and $l(p)=l(q)$, or equivalently $p_{n} \leq_{n}^{*} q_{n}$ for all $n$.

(3) (m-point extension) Let $p$ and $q$ be conditions. Then $p$ is a $m$-point extension of $q$ if and only if $p \leq q$ and $l(p)=l(q)+m$.

(4) $p \leq_{j}^{\sharp} q$ if and only if $p \leq^{*} q$ and $p_{n} \leq_{n}^{* *} q_{n}$ for all $n<j$, that is $l(p)=l(q)$ and $a_{n}^{p}=a_{n}^{q}$ for $n$ such that $l(p) \leq n<j$.

\section{Lemma 4.11.}

(1) Each of $\leq, \leq^{*}, \leq_{j}^{\sharp}$ is a partial ordering of $\mathbb{Q}^{\vec{E}}$. Moreover $\leq^{*}$ refines $\leq, \leq_{0}^{\sharp}=\leq^{*}$, and $\leq_{j+1}^{\sharp}$ refines $\leq_{j}^{\sharp}$.

(2) The poset $\left(\mathbb{Q}^{\vec{E}}, \leq\right)$ is $\kappa_{\omega}^{++}$-c.c.

(3) The ordering $\leq^{*}$ is $\kappa_{l(p)}$-directed closed below the condition $p$.

(4) If $\left\langle r_{n}: n\langle\omega\rangle\right.$ is an $\omega$-sequence of conditions in $\mathbb{Q}^{\vec{E}}$ such that $r_{j+1} \leq_{j}^{\sharp} r_{j}$ then there is a unique weakest condition $r_{\infty}$ such that $r_{\infty} \leq_{j}^{\sharp} r_{j}$ for all $j$.

The last clause of the preceding lemma is reminiscent of the Fusion Lemma and will be used in a very similar way.

Definition 4.12. Let $p$ be a condition where $p_{i}=f_{i}$ for $i<l(p)$ and $p_{i}=\left(a_{i}, A_{i}, f_{i}\right)$ for $i \geq l(p)$. Let $s=\left(\alpha_{l}, \ldots \alpha_{l+m-1}\right)$ where $\alpha_{i} \in A_{i}$ and $l=l(p)$. Then $p+s$ is the condition $q$ such that $q_{j}=p_{j}$ except for $l \leq j<l+m$ where $q_{j}=p_{j}+\alpha_{j}$.

The following lemma follows readily from the corresponding discussion for the basic module in the last section.

Lemma 4.13. If $r \leq p$ then

(1) $r \leq^{*} p+s$ for a unique finite tuple of ordinals $s$.

(2) There is a condition $q$ such that
(a) $r=q+s$.
(b) $q \leq^{*} p$.
(c) $q_{j} \leq_{j 0}^{++} p_{j}$ for $j$ such that $\operatorname{lh}(p) \leq j<\operatorname{lh}(p)+\operatorname{lh}(s)$.

4.4. The generic functions. It is routine to check for every $n$, the set of conditions $p$ with $l(p)>n$ is dense. Given a generic filter $G$, if we define

$$
F_{n}=\bigcup\left\{p_{n}: p \in G, l(p)>n\right\}
$$

then $F_{n}$ is a function from $\rho$ to $\kappa_{n}$. We then define a sequence $\left\langle f_{\alpha}\right.$ : $\alpha<\rho\rangle$ of functions in $\prod_{n} \kappa_{n}$ by setting $f_{\alpha}(n)=F_{n}(\alpha)$. 
These functions are not all distinct, in fact an easy genericity argument shows that $f_{\alpha}$ is identically zero for unboundedly many $\alpha$. However it can be shown that there is an unbounded set $X \subseteq \rho$ such that $\left\langle f_{\alpha}: \alpha \in X\right\rangle$ is strictly increasing in the eventual domination ordering. We will give this argument in Section 5. In particular forcing with $\mathbb{Q}^{\vec{E}}$ adds $\rho$ distinct elements of $\prod_{n} \kappa_{n}$.

4.5. Prikry lemma and related technical facts. The following "Prikry Lemma" is proved in [12] and shows by the usual arguments that forcing with $\mathbb{Q}^{\vec{E}}$ adds no bounded subsets of $\kappa_{\omega}$.

Lemma 4.14. For every sentence $\phi$ of the forcing language and every condition $q \in \mathbb{Q}^{\vec{E}}$ there is $r \leq^{*} q$ such that $r$ decides $\phi$.

We collect some technical facts which are proved by similar arguments to that for the Prikry Lemma, and will be used in proving Theorem 4.19 and Remark 5.4.

Lemma 4.15. Let $D$ be a dense open subset of $\mathbb{Q}^{\vec{E}}$ and let $q \in \mathbb{Q}^{\vec{E}}$. Then there exist an integer $m$ and a condition $t \leq{ }^{*} q$ such that $t+s \in D$ for every $m$-tuple of ordinals s such that $t+s$ is defined.

Proof. let $l={ }_{\text {def }} l(q)$. We will build a sequence of conditions $\left\langle q^{j}: j<\omega\right\rangle$ such that

(1) $q^{0}=q$

(2) $q^{j+1} \leq_{l+j+1}^{\sharp} q^{j}$ for all $j$.

(3) If there is any $r \leq q^{j}$ with $r \in D$ and $l(r)=l+j$, then $q^{j}+s \in D$ for all $j$-tuples $s$ such that $q^{j}+s$ is defined.

Before describing the construction of the sequence $\left\langle q^{j}: j<\omega\right\rangle$, we show that this construction will suffice to prove the Lemma. Given $\left\langle q^{j}: j<\omega\right\rangle$ as above, we may appeal to Lemma 4.11 and choose $t$ such that $t \leq \leq^{*} q^{j}$ for all $j$. Now choose $r \leq t$ with $r \in D$ and say $l(r)=l+m$. Then by construction $t+s \in D$ for every $m$-tuple $s$ such that $t+s$ is defined.

Suppose that we have defined $q^{j}$. Let $q_{n}^{j}=f_{n}$ for $n<l$ and $q_{n}^{j}=$ $\left(a_{n}, A_{n}, f_{n}\right)$ for $n \geq l$.

We enumerate the set of $(j+1)$-tuples $\left(\alpha_{0}, \alpha_{1}, \ldots \alpha_{j}\right)$ with $\alpha_{m} \in A_{l+m}$ for $0 \leq m \leq j$ as $\left\langle s_{i}: i<\kappa_{l+j}\right\rangle$. We then build a chain of conditions $\left\langle r^{i}: i<\kappa_{l+j}\right\rangle$ such that

(1) $r^{0}=q^{j}$.

(2) The sequence $\left\langle r^{i}: i<\kappa_{l+j}\right\rangle$ is $\leq^{*}$-decreasing in $\mathbb{Q}^{\vec{E}}$.

(3) The sequence $\left\langle\left(r^{i}\right)_{n}: i<\kappa_{l}\right\rangle$ is $\leq_{n 0}^{++}$-decreasing in $\mathbb{Q}_{0}^{E^{n}}$ for $l \leq n \leq l+j$. 
(4) If there is any direct extension of $r^{i}+s_{i}$ which lies in $D$, then $r^{i+1}+s_{i}$ is such an extension.

This is possible by Lemmas 4.7 and 4.13 , note in particular we are using the $\kappa_{\omega}^{+}$-closure of the orderings $\leq_{n 0}^{++}$.

We may now find $r^{*}$ such that such that for all $i<\kappa_{l+j}$ we have $r^{*} \leq^{*} r^{i}$, and $\left(r^{*}\right)_{n} \leq_{n 0}^{++}\left(r^{i}\right)_{n}$ for $l \leq n \leq l+j$. The construction guarantees that if there is any $r \leq r^{*}$ with $r \in D$ and $l(r)=l(q)+j+1$ then $r^{*}+s \in D$ for the unique $s$ such that $r \leq^{*} r^{*}+s$.

To finish the construction, we consider the 2-colouring $c$ of $(j+1)$ tuples $s \in \prod_{i=0}^{j} A_{l+i}^{r^{*}}$ in which $c(s)=0$ if $r^{*}+s \notin D$, and $c(s)=1$ if $r^{*}+s \in D$. Appealing to Rowbottom's theorem we may find measure one sets $B_{l+i} \subseteq A_{l+i}^{r^{*}}$ for $0 \leq i \leq j$ such that $c$ is constant on $\prod_{i=0}^{j} B_{l+i}$. Replacing each $A_{l+i}^{r^{*}}$ by $B_{l+i}$, we obtain $q^{j+1} \leq_{l+j+1}^{\sharp} q^{j}$ such that one of the following holds:

- For every $j+1$-tuple $s$ such that $q^{j+1}+s$ is defined, $q^{j+1}+s \in D$.

- For every $j+1$-tuple $s$ such that $q^{j+1}+s$ is defined, $q^{j+1}+s \notin D$.

Corollary 4.16. Let $G$ be $\mathbb{Q}^{\vec{E}}$-generic. Let $A \in V[G]$ be a set of ordinals such that $\omega<c f(A)<\kappa_{\omega}$. Then $A$ has a cofinal subset $B \in V$.

Proof. We recall that $p$ is an $m$-point extension of $q$ when $p \leq q$ and $l(p)=l(q)+m$. Let $\omega<\eta=c f(\eta)<\kappa_{\omega}$ and let $\dot{f}$ name an increasing function from $\eta$ to ON. Let $q$ be an arbitrary condition; extending $q$ if necessary we may as well assume that $l(q)=n$ where $\kappa_{n}>\eta$. By repeated use of Lemma 4.15, we may build a direct extension $q^{*}$ of $q$, such that for every $i<\eta$ there is $m_{i}$ so that every $m_{i}$-point extension of $q^{*}$ decides $\dot{f}(i)$. Find $m$ and an unbounded set $B_{0} \subseteq \eta$ such that $m_{i}=m$ for all $i \in B_{0}$. Let $p$ be an arbitrary $m$-point extension of $q^{*}$, and for each $i \in B_{0}$ let $\gamma_{i}$ be such that $p \Vdash \dot{f}(i)=\gamma_{i}$. Finally let $B=\left\{\gamma_{i}: i \in B_{0}\right\}$.

Corollary 4.17. Let $p$ force that $\dot{\tau}$ is an ordinal and let $m<\omega$. Then there exist a set $B$ of size less than $\kappa_{\omega}$ and a condition $q \leq_{l(p)+m}^{\sharp} p$ such that $q \Vdash \dot{\tau} \in B$.

Proof. Let $D$ be the dense set of conditions which determine the value of $\tau$. We perform a construction just like that of Lemma 4.15 except that we diagonalise over $(m+i)$-tuples for $0 \leq i<\omega$. This produces a condition $q \leq_{l(p)+m}^{\sharp} p$ and a natural number $n$ such that every $n$-step extension of $q$ determines $\dot{\tau}$. Let $B$ be the set of ordinals $\alpha$ such that some $n$-step extension $r$ of $q$ forces $\dot{\tau}$ to equal $\alpha$. 
Corollary 4.18. Let $p$ force that $\dot{f}$ is an $\omega$-sequence of ordinals and let $m<\omega$. Then there are a set $B$ of size $\kappa_{\omega}$ and a condition $q \leq_{l(p)+m}^{\sharp} p$ such that $q \Vdash \operatorname{rge}(\dot{f}) \subseteq B$. In particular $\mathbb{Q}^{\vec{E}}$ preserves $\kappa_{\omega}^{+}$.

Proof. Immediate from Clause 4 of Lemma 4.11 and Corollary 4.17

4.6. Rank-into-rank embeddings. Recall our convention that when $j$ is an elementary embedding we let $\kappa_{0}=\operatorname{crit}(j), \kappa_{n+1}=j\left(\kappa_{n}\right)$ and $\lambda=\sup _{n} \kappa_{n}$. There is a hierarchy of rank-into-rank embeddings which has been studied extensively by Laver [20].

The weakest rank-into-rank embedding axiom asserts the existence of an embedding $j: V_{\lambda} \rightarrow V_{\lambda}$. Given such an embedding $j$ there is a natural way of extending it to an embedding from $V_{\lambda+1} \rightarrow V_{\lambda+1}$ by defining $j(A)=\bigcup_{n} j\left(A \cap V_{\kappa_{n}}\right)$; we can now define a hierarchy of embeddings using the $\Sigma_{n}^{1}$ hierarchy of second-order formulae over the structure $V_{\lambda}$ to measure how elementary the extended $j$ is. In particular Laver [20] has shown that the existence of a $\Sigma_{1}^{1}$ embedding from $V_{\lambda}$ to $V_{\lambda}$ is equivalent to the following large cardinal axiom:

$(*)$ : There is an elementary embedding $j: V \rightarrow M$ such that $V_{\lambda} \subseteq M$.

We will use the following strengthening of this axiom:

$(* *)$ : There is an elementary embedding $j: V \rightarrow M$ such that $V_{\lambda} \subseteq M$, and $\lambda$ is a limit of supercompact cardinals.

Laver [18] has shown (responding to a question by the authors) that if there is a $\Sigma_{3}^{1}$ embedding from $V_{\lambda^{*}}$ to $V_{\lambda^{*}}$ then there is $\lambda<\lambda^{*}$ satisfying $(* *)$.

After these preliminaries we can now state a theorem.

Theorem 4.19. Let GCH and axiom (**) hold. Then there is a generic extension in which $2^{\lambda}=\lambda^{++}$and axiom $(*)$ holds.

Proof. Let $\left\langle\lambda_{n}: n<\omega\right\rangle$ be an increasing sequence of supercompact cardinals with limit $\lambda$. Each $\lambda_{n}$ is $(\lambda+2)$-strong, which is enough to construct a forcing poset $\mathbb{Q}$ as in section 4.3 which adds $\lambda^{++}$subsets of $\lambda$ without adding bounded subsets of $\lambda$. We note in particular that forcing with $\mathbb{Q}$ does not change $V_{\lambda}$.

We will follow a standard line of argument to show that $(* *)$ is preserved by forcing with $\mathbb{Q}$. Given an appropriate embedding $j: V \rightarrow M$ witnessing (**) and $G$ which is $\mathbb{Q}$-generic over $V$, we will find $H \in V[G]$ which is $j(\mathbb{Q})$-generic over $M$ and satisfies an appropriate compatibility condition described below. We will then use the compatibility to define in $V[G]$ an elementary embedding $j: V[G] \rightarrow M[H]$ extending the original $j: V \rightarrow M$, and argue that this embedding witnesses $(* *)$ in $V[G]$. The interested reader will find many further examples 
of this kind of construction in the survey paper by Cummings in the Handbook of Set Theory [1].

Our first task is to show that without loss of generality the embedding $j$ can be assumed to have a special form (in the jargon of inner model theory we are showing that we can take $j$ to be an "ultrapower by a long extender"). Let $j: V \rightarrow M$ with $V_{\lambda} \subseteq M$. Let $X_{0}=\{j(F)(a)$ : $\left.\operatorname{dom}(F) \in V_{\lambda}, a \in V_{\lambda}\right\}$, where we note that $V_{\lambda} \subseteq X_{0}$. Let $\pi: X_{0} \rightarrow M_{0}$ be the Mostowski collapsing map. Standard arguments show that $V_{\lambda} \subseteq$ $M_{0}$, the map $j_{0}={ }_{\text {def }} \pi \circ j$ is an elementary embedding, the embeddings $j$ and $j_{0}$ agree below $\lambda$, and $M_{0}=\left\{j_{0}(F)(a): \operatorname{dom}(F) \in V_{\lambda}, a \in V_{\lambda}\right\}$. The upshot of all this is that replacing $M$ and $j$ by $M_{0}$ and $j_{0}$, we may assume that

$$
M=\left\{j(F)(a): \operatorname{dom}(F) \in V_{\lambda}, a \in V_{\lambda}\right\} .
$$

Let $G$ be a $\mathbb{Q}$-generic filter and let $H$ be the filter on $j(\mathbb{Q})$ generated by the pointwise image $j$ " $G$ of $G$ under $j$. It will suffice to show that $H$ is $j(\mathbb{Q})$-generic over $M$. Since $j$ " $G \subseteq H$, if we attempt to define an extension of our original $j$ onto $V[G]$ by $j: \dot{\tau}^{G} \mapsto j(\dot{\tau})^{H}$ then we get a well-defined and elementary $j: V[G] \rightarrow M[H]$. Since $V_{\lambda}=V_{\lambda}^{V[G]}$, this extended embedding will witness that $(*)$ holds in $V[G]$.

Let $D \in M$ be a dense open subset of $j(\mathbb{Q})$. We may assume that $D=j(F)(a)$ where there is $n$ such that $\operatorname{dom}(F)=V_{\lambda_{n}}$, and $F(x)$ is a dense open subset of $\mathbb{Q}$ for every $x$. We will show that there is a dense set of conditions $q \in \mathbb{Q}$ such that $j(q) \in D$. We fix an enumeration of $V_{\lambda_{n}}$ as $\left\langle x_{i}: i<\lambda_{n}\right\rangle$.

Let $p \in \mathbb{Q}$ be arbitrary. Find $q \leq p$ such that the length of $q$ is greater than $n$, so that below $q$ the direct extension relation $\leq^{*}$ is $\lambda_{n}^{+}$closed. By the analysis of dense sets in Lemma 4.15 we may find a $\leq^{*}$-decreasing sequence of conditions $\left\langle r_{i}: i<\lambda_{n}\right\rangle$ and a sequence of natural numbers $\left\langle m_{i}: i<\lambda_{n}\right\rangle$, such that $r_{i} \leq^{*} p$ and for every $i$ every $m_{i}$-step extension of $r_{i}$ is in $F\left(x_{i}\right)$.

Let $r_{\infty}$ be a lower bound for the sequence $\left\langle r_{i}: i\left\langle\lambda_{n}\right\rangle\right.$ and let $m_{\infty}=j(M)(a)$, where $M: V_{\lambda_{n}} \rightarrow \omega$ is given by $M: x_{i} \mapsto m_{i}$. Let $s \leq r_{\infty}$ be any $m_{\infty}$-step extension of $r_{\infty}$. Then by elementarity $j(s) \in j(F)(a)=D$, and we are done.

Remark 4.20. Since the cardinals $\lambda_{n}$ are fully supercompact in the ground model, we could have chosen longer extenders in the proof of Theorem 4.19 and made the power set of $\lambda$ arbitrarily large. 
Remark 4.21. There would be no difficulty in preserving the existence of an elementary $j: V_{\lambda} \rightarrow V_{\lambda}$ while making $2^{\lambda}$ arbitrarily large, since $V_{\lambda}$ is not changed by the forcing construction of Theorem 4.19.

Remark 4.22. Moti Gitik [10] has observed that one should be able to lower the hypothesis to $(*)$ (which will clearly be optimal) by using his technology of "extender based forcing with short extenders"[12].

Remark 4.23. One of the strongest axioms of the type we have been discussing is the axiom asserting the existence of an embedding $j$ : $L\left(V_{\lambda+1}\right) \rightarrow L\left(V_{\lambda+1}\right)$. Hugh Woodin has developed a detailed structure theory for $L\left(V_{\lambda+1}\right)$ under this hypothesis, analogous to the structure theory for $L(\mathbb{R})$ under $A D$. Woodin's theory gives [29] a consistency proof for the assertion "there is $j: V_{\lambda+1} \rightarrow V_{\lambda+1}$ with $2^{\lambda}>\lambda^{+}$".

\section{A NON-REFLECTING STATIONARY SET}

We finish by recording a remark on a construction of Sharon [24]. Sharon showed that if $\mathbb{Q}$ is an extender-based forcing of the sort described in section 4.3 then $\kappa_{\omega}^{+} \cap \operatorname{cof}(\omega)$ contains a non-reflecting stationary set. In some of the authors' joint work with Magidor [4], we discovered a number of constructions in which non-reflecting or noncompact objects are constructed from PCF-theoretic scales. In this section we will show that Sharon's construction fits nicely into this framework.

Let $\vec{f}=\left\langle f_{i}: i<\mu^{+}\right\rangle$be a good scale of length $\mu^{+}$in $\prod_{i<\omega} \mu_{i}$, where the $\mu_{i}$ are regular cardinals and are increasing and cofinal in the singular cardinal $\mu$. Let $S(\vec{f})$ be the subset of $\mu^{+} \cap \operatorname{cof}(\omega)$ consisting of those $\beta$ of cofinality $\omega$ such there is no $m<\omega$ and $B \subseteq \beta$ unbounded in $\beta$ so that $\left\langle f_{\gamma}(n): \gamma \in B\right\rangle$ is strictly increasing for all $n \geq m$.

Clearly if $c f(\alpha)>\omega$ and $A, m$ witness the goodness property at $\alpha$ then $\beta \notin S(\vec{f})$ for every $\beta<\alpha$ such that $\beta=\sup (A \cap \beta)$. It follows that if $C$ is a club subset of $\mu^{+}$such that for every $\alpha \in C \cap \operatorname{cof}(>\omega)$ there exist $A$ and $m$ as above, then $S(\vec{f}) \cap C$ is non-reflecting; so if $S(\vec{f})$ happens to be stationary (which may or may not be the case) then we have a construction for a non-reflecting stationary set.

Note that the construction of the last paragraph does not need that $\left\langle f_{\alpha}: \alpha<\mu^{+}\right\rangle$is cofinal in $\prod_{n} \mu_{n} /$ finite, merely that it is an increasing sequence which is good at almost all $\alpha \in \mu^{+} \cap \operatorname{cof}(>\omega)$. Shelah's Trichotomy Theorem ([3]) can be used to show that any such increasing sequence has an exact upper bound $g$ such that $\lim _{n \rightarrow \infty} c f(g(n))=\mu$; if $\left\langle\bar{\mu}_{n}: n<\omega\right\rangle$ enumerates $\{\operatorname{cf}(g(n))\}$ in increasing order then it is easy 
to "collapse" our original scale and get a good scale $\left\langle g_{\alpha}: \alpha<\mu^{+}\right\rangle$in $\prod_{n} \bar{\mu}_{n} /$ finite.

Now let $\mathbb{Q}=\mathbb{Q}^{\vec{E}}$ be a forcing poset of the sort described in section 4.3 , where $\rho \geq \kappa_{\omega}^{++}$. Let $G$ be $\mathbb{Q}$-generic and let $\left\langle f_{\alpha}: \alpha<\rho\right\rangle$ be the functions in $\prod_{n} \kappa_{n}$ added by $\mathbb{Q}$, as described in subsection 4.4. It is not the case that the $f_{\alpha}$ are increasing modulo finite. An easy density argument shows that $f_{\alpha}$ is identically zero for cofinally many $\alpha<\rho$. However we can identify a subsequence that is increasing modulo finite.

Recall that conditions in $\mathbb{Q}$ have the form

$$
p=\left\langle f_{0}^{p}, f_{1}^{p}, \ldots f_{l-1}^{p},\left(a_{l}^{p}, A_{l}^{p}, f_{l}^{p}\right),\left(a_{l+1}^{p}, A_{l+1}^{p}, f_{l+1}^{p}\right), \ldots\right\rangle
$$

where $a_{n}^{p} \subseteq \rho$ with $\left|a_{n}^{p}\right|<\kappa_{n}$ and $a_{n}^{p} \subseteq a_{n+1}^{p}$. Define $X_{p}=\bigcup_{i \geq l} a_{l}^{p}$ for each $p$, and then in $V[G]$ define $X=\bigcup_{p \in G} X_{p}$. The following facts are routine:

(1) For every $\alpha<\rho, f_{\alpha} \notin V$ if and only if $\alpha \in X$.

(2) $X \cap \kappa_{\omega}^{+}$is unbounded in $\kappa_{\omega}^{+}$.

(3) If we enumerate $X \cap \kappa_{\omega}^{+}$in increasing order as $\left\langle\alpha_{j}: j<\kappa_{\omega}^{+}\right\rangle$and define $h_{j}=f_{\alpha_{j}}$, then $\left\langle h_{j}: j<\kappa_{\omega}^{+}\right\rangle$is an increasing sequence in $\prod_{n} \kappa_{n} /$ finite.

(4) $p \Vdash \alpha \in \dot{X}$ if and only if $\alpha \in X_{p}$.

(5) If $\beta<\rho$ with $\operatorname{cf}(\beta)>\omega$, then $p \Vdash \beta=\sup (\dot{X} \cap \beta)$ if and only if there is $m<\omega$ such that $\beta=\sup \left(a_{m}^{p} \cap \beta\right)$.

Note that the last claim fails for $\beta$ with $\operatorname{cf}(\beta)=\omega$. In that case there are two ways that a condition $p$ may force that $\beta=\sup (\dot{X} \cap \beta)$; either there is $m$ with $\beta=\sup \left(a_{m}^{p} \cap \beta\right)$, or no such $m$ exists and the sequence $\left\langle\sup \left(a_{n}^{p} \cap \beta\right): \operatorname{lh}(p) \leq n<\omega\right\rangle$ is cofinal in $\beta$.

Now we start to analyse the sequence $\left\langle h_{j}: j<\kappa_{\omega}^{+}\right\rangle$.

Lemma 5.1. For every $\beta \in \kappa_{\omega}^{+} \cap \operatorname{cof}(>\omega)$ there exist $A \subseteq \beta$ unbounded and $m<\omega$ such that $\left\langle h_{j}(n): j \in A\right\rangle$ is strictly increasing for all $n \geq m$.

Proof. Let $\beta<\kappa_{\omega}^{+}$with $\operatorname{cf}(\beta)>\omega$ and define $\gamma=\sup \left(X \cap \alpha_{\beta}\right)$. Note that $X \cap \gamma=\left\{\alpha_{i}: i<\beta\right\}$, and $\gamma=\sup (X \cap \gamma)=\sup _{i<\beta} \alpha_{i}$. In particular $\operatorname{cf}(\gamma)=\operatorname{cf}(\beta)>\omega$. Choose $p \in G$ such that $p \Vdash \gamma=$ $\sup (X \cap \gamma)$, and fix $m$ such that $\gamma=\sup \left(a_{m}^{p} \cap \gamma\right)$. Let $A^{*}=a_{m}^{p} \cap \gamma$, so that $A^{*} \subseteq X \cap \gamma$ with $\sup \left(A^{*}\right)=\gamma$.

For every $n \geq m$ we have that $A^{*} \subseteq a_{n}^{p}$, and also every $\nu \in A_{p}^{n}$ is sorted for $a_{n}^{p}$. It follows easily from the second clause in Definition 4.3 and the definition of the functions $f_{\alpha}$ that

$$
p \Vdash\left\langle f_{\alpha}(n): \alpha \in A^{*}\right\rangle \text { is strictly increasing }
$$


for every $n \geq m$. If we now let $A=\left\{i<\beta: \alpha_{i} \in A^{*}\right\}$ then $A$ is unbounded in $\beta$, and $\left\langle h_{i}(n): i \in A\right\rangle$ is strictly increasing for all $n \geq m$.

The preceding lemma says that $\vec{h}$ is good at every $\beta \in \kappa_{\omega}^{+} \cap \operatorname{cof}(>\omega)$, so by the discussion at the start of this section $S(\vec{h}) \cap \beta$ is non-stationary for every such $\beta$. The proof of the following lemma mildly simplifies Sharon's argument that $S(\vec{h})$ is stationary.

Lemma 5.2. In $V[G]$ the set $S(\vec{h})$ is stationary.

Proof. Let $p \in \mathbb{Q}$ and let $\dot{C}$ be a name for a club subset of $\kappa_{\omega}^{+}$. Let $\theta$ be a very large regular cardinal, let $<_{\theta}$ be a wellordering of $H_{\theta}$ and let $N \prec\left(H_{\theta}, \in,<_{\theta}\right)$ be such that $|N|=\kappa_{\omega} \subseteq N, N$ contains everything relevant, and $\operatorname{cf}(\beta)=\omega$ where $\beta={ }_{\text {def }} N \cap \kappa_{\omega}^{+}$.

Using Corollary 4.17 and the facts that $N$ is an elementary substructure and $c f(\beta)=\omega$, we build a sequence of conditions $\left\langle p_{n}: n<\omega\right\rangle$ and a sequence of ordinals $\left\langle\beta_{n}: n<\omega\right\rangle$ such that

(1) $p_{0}=p, \beta_{0}=0$.

(2) $p_{n} \in N \cap \mathbb{Q}$ and $\beta_{n} \in N \cap \kappa_{\omega}^{+}$.

(3) $p_{n+1} \leq_{\operatorname{lh}(p)+n}^{\sharp} p_{n}$.

(4) $p_{n+1}$ forces that the $\beta_{n}^{\text {th }}$ point of $X$ is less than $\beta_{n+1}$.

(5) $p_{n+1}$ forces that $\min \left(\dot{C} \backslash \beta_{n}\right)$ is less than $\beta_{n+1}$.

(6) $\left\langle\beta_{n}: n<\omega\right\rangle$ is increasing and cofinal in $\beta$.

By clause 4 of Lemma 4.11 , we find a condition $p_{\infty}$ such that $p_{\infty}$ is a lower bound for the $p_{n}$ in the strong sense that $p_{\infty} \leq_{\operatorname{lh}(p)+n}^{\sharp} p_{n}$ for all $n$. In particular if $\eta_{n}=\operatorname{def} \sup \left(\left(a_{\operatorname{lh}(p)+n}^{p_{\infty}} \cup \operatorname{dom}\left(f_{\operatorname{lh}(p)+n}^{p_{\infty}}\right)\right) \cap \beta\right)$, then $\eta_{n}<\beta$.

Now we define $r \leq^{*} p_{\infty}$ such that for all $n$

$$
\operatorname{dom}\left(f_{\operatorname{lh}(p)+n}^{r}\right)=\operatorname{dom}\left(f_{\operatorname{lh}(p)+n}^{p_{\infty}}\right) \cup\left[\eta_{n}, \beta\right),
$$

and $f_{\operatorname{lh}(n)+p}^{r}$ is identically zero on the interval $\left[\eta_{n}, \beta\right)$. Then $r$ forces that

(1) $\beta \in C$.

(2) ot $(X \cap \beta)=\beta$.

(3) If $B$ is any unbounded subset of $X \cap \beta$ and $n<\omega$, then $\left\langle f_{\gamma}(n)\right.$ :

$\gamma \in B\rangle$ is eventually zero.

The conclusion follows.

We did not need to know the exact upper bound for the scale $\left\langle h_{j}\right.$ : $j\left\langle\kappa_{\omega}^{+}\right\rangle$in this argument. However it is a natural question to ask what this upper bound is, especially in the light of the results of Section 2. 
We finish by sketching a computation of this exact upper bound. We speculate that a further PCF analysis may shed more light on singular cardinal combinatorics in the generic extension by $\mathbb{Q}$, but do not pursue this here.

We recall that for each $n$ we generated a sequence of measures $\left\langle E_{\alpha}^{n}\right.$ : $\alpha<\rho\rangle$ by $E_{\alpha}^{n}=\left\{X \subseteq \kappa_{n}: \alpha \in j_{n}(X)\right\}$, where $j_{n}: V \rightarrow M_{n}$ has critical point $\kappa_{n}$ and all bounded subsets of $\rho$ are in $M_{n}$. For each $n$ we have that $E_{\alpha}^{n} \leq_{R K} E_{\beta}^{n}$ if and only if there is $f: \kappa_{n} \rightarrow \kappa_{n}$ with $j_{n}(f)(\beta)=\alpha$, and any such function $f$ Rudin-Keisler projects $E_{\beta}$ to $E_{\alpha}$. We fixed $\pi_{\alpha \beta}^{n}$ such that $j_{n}\left(\pi_{\alpha \beta}^{n}\right)(\beta)=\alpha$ whenever $E_{\alpha}^{n} \leq_{R K} E_{\beta}^{n}$.

Claim 5.3. In $V[G]$ there is $\gamma \in X$ such that $\kappa_{\omega}^{+}<\gamma$ and $E_{\kappa_{\omega}^{+}}^{n} \leq{ }_{R K} E_{\gamma}^{n}$ for all $n$.

Proof. Let $p$ be an arbitrary condition. We will show how to find $q \leq p$ which forces a suitable $\gamma$ into $X$. Let $\Phi: O n \rightarrow O n$ be some absolutely definable function such that for every infinite regular cardinal $\mu, \Phi \uparrow \mu$ is a $\mu$-to-one map from $\mu$ to $\mu$; such a function is easily defined using Gödel's pairing function. Choose $\gamma<\kappa_{\omega}^{++}$which does not appear in $p$, and is such that $\kappa_{\omega}^{+}<\gamma$ and $\Phi(\gamma)=\kappa_{\omega}^{+}$. Since $\Phi$ has an absolute definition it is easy to check that $j_{n}\left(\Phi\left\lceil\kappa_{n}\right)(\gamma)=\Phi(\gamma)=\kappa_{\omega}^{+}\right.$for all $n$. Now we may extend $p$ to force $\gamma$ into $X$.

Working in $V[G]$, let $\gamma$ be the least ordinal as in the last claim, and define a function $h \in \prod_{n} \kappa_{n}$ by $h(n)=\pi_{\kappa_{\omega}^{+}, \gamma}^{n}\left(f_{\gamma}(n)\right)$. The intuition behind this definition is that $\kappa_{\omega}^{+}$may not be an element of $X$, but that if it were then this is (mod finite) the function which would appear as $f_{\kappa_{\omega}^{+}}$.

We claim that $h$ is an exact upper bound for $\left\langle h_{j}: j\left\langle\kappa_{\omega}^{+}\right\rangle\right.$in $V[G]$. Start by noting that since $X \cap \kappa_{\omega}^{+}$is unbounded in $\kappa_{\omega}^{+},\left\{f_{\delta}: \delta \in\right.$ $\left.X \cap \kappa_{\omega}^{+}\right\}=\left\{h_{j}: j<\kappa_{\omega}^{+}\right\}$.

Let $p$ be a condition forcing that $\dot{g}(n)<\dot{h}(n)$ for all $n$. Extending $p$ if necessary, we may assume that $p$ determines the value of $\gamma$, in particular $p$ forces that $\gamma$ is in $X$. By the same sort of diagonalisation argument as in Lemma 4.15) we may build a condition $q \leq^{*} p$ and a sequence of functions $\left\langle H_{n}: \operatorname{lh}(q) \leq n<\omega\right\rangle$, such that $\operatorname{dom}\left(H_{n}\right)=A_{n}^{q}$ and $q$ forces that $\dot{g}(n)=H_{n}\left(f_{\max a_{n}^{q}}(n)\right)$. Extending $q$ further we may also assume that $\gamma \in a_{l h(q)}^{q}$.

Let $\rho_{n}=\max a_{n}^{q}$, and let $\beta_{n}=j_{n}\left(H_{n}\right)\left(\rho_{n}\right)$. We claim that $\beta_{n}<$ $\kappa_{\omega}^{+}$. To see this note that $q$ forces that $f_{\gamma}(n)=\pi_{\gamma \rho_{n}}\left(f_{\rho_{n}}(n)\right)$, and so it forces that $h(n)=\pi_{\kappa_{\omega}^{+} \gamma} \circ \pi_{\gamma \rho_{n}}\left(f_{\rho_{n}}(n)\right)$. Furthermore $q$ forces that $\dot{g}(n)=H_{n}\left(f_{\rho_{n}}(n)\right)$, and the possible values of $f_{\rho_{n}}(n)$ are exactly the elements of $A_{n}^{q}$. Finally $q$ forces that $\dot{g}(n)<\dot{h}(n)$. So for all $\nu \in A_{n}^{q}$, 
$H_{n}(\nu)<\pi_{\kappa_{\omega}^{+} \gamma} \circ \pi_{\gamma \rho_{n}}(\nu)$; since $A_{\nu}^{q} \in E_{\rho_{n}}^{n}$ it follows from the definitions that $\beta_{n}=j_{n}\left(H_{n}\right)\left(\rho_{n}\right)<\kappa_{\omega}^{+}$as claimed.

To finish we find $r \leq q$ and $\delta$ such that $\sup _{l h(q) \leq n<\omega} \beta_{n}<\delta<\kappa_{\omega}^{+}$, and $r \Vdash \delta \in \dot{X}$. By similar arguments to those in the previous paragraph, it is routine to check that $r \Vdash \dot{g}<^{*} f_{\delta}$. This concludes the proof that $h$ is an exact upper bound.

We have proved that Sharon's construction of a non-reflecting stationary set in [24] can be presented as follows.

Remark 5.4. If $\mathbb{Q}$ is an extender based forcing of the sort described in section 4.3 , then in the generic extension

(1) There is a good scale of length $\kappa_{\omega}^{+}$.

(2) The non-reflecting set constructed from that good scale is stationary.

\section{REFERENCES}

1. J. Cummings, Iterated forcing and elementary embeddings, To appear in the Handbook of Set Theory.

2. __ Collapsing successors of singulars, Proceedings of the American Mathematical Society 125 (1997), no. 9, 2703-2709.

3. Notes on singular cardinal combinatorics, Notre Dame Journal of Formal Logic 46 (2005), no. 3, 251-282.

4. J. Cummings, M. Foreman, and M. Magidor, Squares, scales and stationary reflection, Journal of Mathematical Logic 1 (2001), no. 1, 35-98.

5. _ The non-compactness of square, The Journal of Symbolic Logic 68 (2003), no. 2, 637-643.

6. Canonical structure in the universe of set theory. I, Annals of Pure and Applied Logic 129 (2004), no. 1-3, 211-243.

7. _ Canonical structure in the universe of set theory. II, Annals of Pure and Applied Logic 142 (2006), no. 1-3, 55-75.

8. M. Foreman, Some problems in singular cardinals combinatorics, Notre Dame Journal of Formal Logic 46 (2005), no. 3, 309-322.

9. M. Foreman and M. Magidor, A very weak square principle, Journal of Symbolic Logic 62 (1997), no. 1, 175-196.

10. M. Gitik, Personal communication.

11. , Prikry-Type Forcings, To appear in the Handbook of Set Theory.

12. Blowing up power of a singular cardinal-wider gaps, Annals of Pure and Applied Logic 116 (2002), no. 1-3, 1-38.

13. M. Gitik and M. Magidor, Extender based forcings, The Journal of Symbolic Logic 59 (1994), no. 2, 445-460.

14. M. Gitik and A. Sharon, On SCH and the approachability property, Proceedings of the American Mathematical Society 136 (2008), no. 1, 311-320.

15. T. Jech, On the cofinality of countable products of cardinal numbers, A tribute to Paul Erdős, Cambridge Univ. Press, Cambridge, 1990, pp. 289-305.

16. Akihiro Kanamori, The Higher Infinite, second ed., Springer Monographs in Mathematics, Springer-Verlag, Berlin, 2003. 
17. K. Kunen, Elementary embeddings and infinitary combinatorics, The Journal of Symbolic Logic 36 (1971), 407-413.

18. R. Laver, Personal communication.

19. _ Making the supercompactness of $\kappa$ indestructible under $\kappa$-directed closed forcing, Israel Journal of Mathematics 29 (1978), no. 4, 385-388.

20. __ Implications between strong large cardinal axioms, Annals of Pure and Applied Logic 90 (1997), no. 1-3, 79-90.

21. M. Magidor, On the singular cardinals problem. I, Israel Journal of Mathematics 28 (1977), no. 1-2, 1-31.

22. M. Magidor and S. Shelah, When does almost free imply free? (For groups, transversals, etc.), Journal of the American Mathematical Society 7 (1994), no. 4, 769-830.

23. I. Neeman, Aronszajn trees and failure of the singular cardinal hypothesis, To appear in the Journal of Mathematical Logic.

24. A. Sharon, Weak squares, scales, stationary reflection and the failure of $\mathrm{SCH}$, Ph.D. thesis, Tel Aviv University, 2005.

25. S. Shelah, Whitehead groups may be not free, even assuming CH. I, Israel Journal of Mathematics 28 (1977), no. 3, 193-204.

26. _ Whitehead groups may not be free, even assuming CH. II, Israel Journal of Mathematics 35 (1980), no. 4, 257-285.

27. D. Sinapova, A model for a very good scale and a bad scale, Journal of Symbolic Logic 73 (2008), no. 4, 1361-1372.

28. R. M. Solovay, Strongly compact cardinals and the GCH, Proceedings of the Tarski Symposium (Proc. Sympos. Pure Math., Vol. XXV, Univ. California, Berkeley, Calif., 1971) (Providence, R.I.), American Mathematical Society, 1974, pp. 365-372.

29. W. H. Woodin, Personal communication.

Mathematical Sciences Department, Carnegie Mellon University, Pittsburgh PA 15215, USA

E-mail address: jcumming@andrew.cmu.edu

Department of Mathematics, University of California, Irvine, Irvine CA 92697, USA

E-mail address: mforeman@math.uci.edu 